\title{
Primate Lentivirus Group
}

National Cancer Institute

\section{Source}

National Cancer Institute. Primate Lentivirus Group. NCI Thesaurus. Code C117716.

A non-taxonomic grouping of lentivuruses that infect humans, apes, monkeys, lemurs,

lorises, and tarsiers. 